\title{
Diffusion-weighted imaging in relation to morphology on dynamic contrast enhancement MRI: the diagnostic value of characterizing non-puerperal mastitis
}

\author{
Lina Zhang ${ }^{1}$ - Jiani $\mathrm{Hu}^{2}$ • Nicholas Guys ${ }^{2}$ - Jinli Meng ${ }^{3}$ - Jianguo Chu ${ }^{1}$. \\ Weisheng Zhang ${ }^{1} \cdot$ Ailian Liu ${ }^{1}$ - Shaowu Wang ${ }^{4} \cdot$ Qingwei Song $^{1}$
}

Received: 6 February 2017 / Revised: 10 August 2017 / Accepted: 22 August 2017 / Published online: 27 September 2017

(C) The Author(s) 2017. This article is an open access publication

\begin{abstract}
Objectives To demonstrate the value of diffusion-weighted imaging (DWI) in the characterisation of mastitis lesions.

Methods Sixty-one non-puerperal patients with pathologically confirmed single benign mastitis lesions underwent preoperative examinations with conventional MRI and axial DWI. Patients were categorised into three groups: (1) periductal mastitis (PDM), (2) granulomatous lobular mastitis (GLM), and (3) infectious abscess (IAB). Apparent diffusion coefficient (ADC) values of each lesion were recorded. A one-way ANOVA with logistic analysis was performed to compare ADC values and other parameters. Discriminative abilities of DWI modalities were compared using the area under the receiver operating characteristic curve (AUC). $P<0.05$ was considered statistically significant.
\end{abstract}

Lina Zhang and Jiani Hu contributed equally to this work, they are co-first authors.

Ailian Liu

alya211@163.com

Shaowu Wang

wsw_2003@163.com

1 Department of Radiology, First Affiliated Hospital of Dalian Medical University, 222 Zhongshan Road, Xigang, Dalian, Liaoning 116011, China

2 Department of Radiology, Wayne State University, 540 East Canfield Street, Detroit, MI 48201, USA

3 Department of Radiology, Chengban Branch of West China Hospital, 37 Guoxue Alley, Wuhou, Chengdu, Sichuan 610041, China

4 Department of Radiology, Second Affiliated Hospital of Dalian Medical University, 467 Zhongshan Road, Shahekou, Dalian, Liaoning 116023, China
Results ADC values differed significantly among the three groups $(P=0.003)$ as well as between PDM and IAB and between PDM and GLM. The distribution of non-mass enhancement on dynamic contrast-enhanced (DCE) MRI differed significantly among the three groups $(P=0.03)$ but not between any two groups specifically. There were no differences in lesion location, patient age, $\mathrm{T}_{2} \mathrm{WI}$ or DWI signal intensity, enhancement type, non-mass internal enhancement, or mass enhancement characteristics among the three groups. Conclusions ADC values and the distribution of non-mass enhancement are valuable in classifying mastitis subtypes.

Key points

- Mastitis subtypes exhibit different characteristics on DWI and DCE MRI.

- ADC values are helpful in isolating PDM from other mastitis lesions.

- Distribution of non-mass enhancement also has value in comparing mastitis subtypes.

Keywords mastitis - granulomatous mastitis · abscess · diffusion magnetic resonance imaging $\cdot$ image enhancement

\author{
Abbreviations \\ MRI magnetic resonance imaging \\ DWI diffusion-weighted imaging \\ DCE dynamic contrast-enhanced \\ ADC apparent diffusion coefficient \\ AUC area under the receiver operating characteristic curve
}

\section{Introduction}

Mastitis is primarily defined as infectious (usually bacterial) or non-infectious inflammation of breast tissue [1].. Non- 
puerperal mastitis describes inflammatory lesions of the breast that occur unrelated to pregnancy and breastfeeding [2]. Currently, non-puerperal mastitis accounts for approximately $4-5 \%$ of benign breast lesions, but its incidence is increasing, especially in developing countries [3]. For example, nonpuerperal mastitis represents approximately $2-5 \%$ of all breast lesions in China, as compared to $0.3-1.9 \%$ of all breast lesions globally $[3,4]$.

Non-infectious non-puerperal mastitis is subclassified as periductal mastitis (PDM) or granulomatous lobular mastitis (GLM), while infectious non-puerperal mastitis most commonly manifests as an infectious abscess (IAB). The most common pathological characteristics of PDM, GLM, and $\mathrm{IAB}$ are as follows: PDM: greyish discharge exuding from dilated lactiferous ducts, resulting in plasma cell-induced inflammation; GLM: chronic, non-caseating granulomatous lobulitis; and IAB: accumulation of pus in a localised region of the breast [5].

Diffusion-weighted imaging (DWI) is an alternative, noncontrast functional magnetic resonance imaging (MRI) technique that is sensitive to the motion of free water molecules in biological tissues, which can reflect the histological characteristics of various lesions [9-12]. DWI often complements conventional MRI data in differentiating mastitis from various cancers and has potential value in classifying various cancer types as well [13-15]. The apparent diffusion coefficient (ADC), a quantitative parameter derived from DWI, has been shown to help differentiate between tumour subtypes $[10,16]$. An anomalous variation in the ADC value may indicate changes such as oedema, cysts, bleeding, thick content, or necrosis $[10,13,14]$.

However, to date, few DWI studies have focused specifically on mastitis lesions, and there currently exists in the literature a paucity of studies examining the DWI and dynamic contrast-enhanced (DCE) MRI characteristics of the different subtypes of mastitis $[13,14]$. To ensure optimal patient outcomes, it is important to classify non-puerperal mastitis before administering therapies. For this reason, our study sought to determine the feasibility of using DWI combined with DCE MRI in evaluating PDM, GLM, and IAB. Utilising DWI and DCE MRI, our aim was to better characterise non-puerperal mastitis lesions and help reduce the interval between definitive diagnosis and administration of specific therapies.

\section{Materials and methods}

\section{Patients and lesions}

This retrospective study was performed from January 2010 to August 2015. Initially, seventy-five female patients underwent pathological assessments and MRI examinations for confirmation of non-puerperal mastitis. Fourteen of these 75 patients were excluded due to incomplete DCE or DWI sequences (as a result of an informatics failure in archiving image scans, including motion artefacts). Thus, 61 female patients with unilateral mastitis were enrolled in this study, assessed using DCE and DWI MRI scans, and categorised into three sub-groups based on the type of mastitis lesion (17 PDM: mean age $=44.47 \pm 14.68 ; 32$ GLM: mean age $=$ $41.34 \pm 12.71 ; 12$ IAB: mean age $=41.33 \pm 18.79$ ). In 51 patients, a pathological diagnosis was made using percutaneous biopsies; in the other 10 patients, a pathological diagnosis was confirmed with surgery. All mastitis lesions were unilateral, and the majority occupied multiple quadrants within the affected breast. The mean age of clinical presentation for each group (i.e. PDM, GLM, and IAB) was consistent with previous reports $[4,17]$.

\section{Imaging protocols}

All patients were imaged using a 3.0T whole-body MR scanner (Signa Excite HDxt, General Electric Healthcare, Milwaukee, WI, USA) with a dedicated eight-channel bilateral phase-array breast coil. Each patient was placed in prone position without compression. Conventional MR scanning sequences included sagittal fat-saturation FSE $\mathrm{T}_{2} \mathrm{WI}$ (repetition time/echo time $[\mathrm{TR} / \mathrm{TE}]=2600 \mathrm{~ms} / 85 \mathrm{~ms}$; slice thickness $=5 \mathrm{~mm}$; number of excitations $[\mathrm{NEX}]=2$; matrix size $=288 \times 224$ zero-filled to $512 \times 256$; field of view $[\mathrm{FOV}]=20 \mathrm{~cm} \times 20 \mathrm{~cm})$ and axial short tau inversion recovery (STIR) sequence $(\mathrm{TR} / \mathrm{TE}=4450$ $\mathrm{ms} / 68 \mathrm{~ms}$; inversion time $[\mathrm{TI}]=210 \mathrm{~ms}$; slice thickness $=5$ $\mathrm{mm} ; \mathrm{NEX}=1$; matrix size $=256 \times 160$ zero-filled to $256 \times 256$; FOV $=40 \mathrm{~cm} \times 40 \mathrm{~cm}$ ). DWI was acquired for both breasts in the axial plane with echo planar imaging $(\mathrm{TR} / \mathrm{TE}=8000 \mathrm{~ms} / 90$ $\mathrm{ms}$; slice thickness $=3 \mathrm{~mm} ; \mathrm{NEX}=6$; matrix size $=96 \times 128$ zero-filled to $128 \times 256$; FOV $=30 \mathrm{~cm} \times 30 \mathrm{~cm} ; \mathrm{b}=0$ and 800 $\mathrm{s} / \mathrm{mm}^{2}$ ) before contrast agent injection. One pre- and seven post-contrast images were acquired using an axial 3D T1weighted VIBRANT (Volume Imaging for Breast Assessment) sequence with fat saturation: $\mathrm{TR} / \mathrm{TE}=3.7 \mathrm{~ms} /$ $2.1 \mathrm{~ms} ; \mathrm{TI}=12 \mathrm{~ms}$; flip angle $[\mathrm{FA}]=10^{\circ}$; slice thickness $=$ $2.2 \mathrm{~mm} ; \mathrm{NEX}=1$; matrix size $=320 \times 320$ zero-filled to $512 \times$ 512 ; FOV $=32 \mathrm{~cm} \times 32 \mathrm{~cm}$. Parallel imaging of autocalibrating reconstruction for cartesian sampling (ARC) was employed. The first post-contrast acquisition was started 25 sec after contrast injection, and the remaining images were acquired every $57 \mathrm{sec}$. The contrast agent (Gadodiamide) was administrated with a dosage of $0.1 \mathrm{mmol} / \mathrm{kg}$ body weight and a mean volume of $15 \pm 3 \mathrm{~mL}$ for each patient. Contrast was administered at a rate of $2.0 \mathrm{~mL} / \mathrm{s}$ using a dedicated power injector.

\section{Imaging analysis}

MRI results were interpreted independently by two radiologists, both with more than ten years of diagnostic experience 
in breast imaging. A diagnostic consensus was reached following a discussion of the images. All data was transferred to a GE workstation (Advantage Windows 4.5, General Electric, Madison, WI, USA). DCE morphology of each lesion was assessed in initial enhancement phases (90 s) according to the American College of Radiology (ACR) breast imagingreporting and data system (BI-RADS) Breast MRI Lexicon ( $5^{\text {th }}$ edition, 2013) [18]. The region of interest (ROI) was obtained manually on the DWI with hyperintensity signal, ideally without necrotic or cystic components. When DWI could not clearly distinguish a mastitis lesion from a necrotic or cystic lesion (often because of its poor spatial resolution), DCE image was used to help to draw the ROI, which corresponding with the most enhanced part of the lesion on DCE. The mean value of three ROI measurements was obtained as the final ADC value.

\section{Statistical analysis}

A one-way ANOVA and post-hoc SNK test were used for analysing ADC values among the three groups (PDM, GLM, and IAB) and between any two groups, respectively. The discriminative abilities of ADC values in arriving at a differential diagnosis were compared using receiver operating characteristic (ROC) curves and the area under the ROC curve (AUC). Conventional MRI and DCE MRI morphologies and clinical information, such as age of presentation and lesion location, were analysed using a chi-square test or Fisher's exact test. Analysis was performed using SPSS software (SPSS Version 17, SPSS Inc., Chicago, IL, USA). $P<0.05$ was considered indicative of a statistically significant difference among the groups. $P<0.0167$ indicated a significant difference between any two groups compared using a chisquare test. Results of the SNK test did not provide $p$-values.

\section{Results}

The mean ADC values for the three groups were as follows: PDM: $(1.558 \pm 0.34) \times 10^{-3} \mathrm{~mm}^{2} / \mathrm{s}$; GLM: $(1.244 \pm 0.32) \times$ $10^{-3} \mathrm{~mm}^{2} / \mathrm{s}$; and IAB: $(1.105 \pm 0.44) \times 10^{-3} \mathrm{~mm}^{2} / \mathrm{s}$. One-way ANOVA revealed a significant difference in ADC values among the three groups $(\mathrm{F}=6.589, P=0.003)$. Post-hoc SNK test results are shown in Table 1 . There was a significant difference between PDM and GLM and between PDM and IAB. GLM and IAB did not differ significantly in ADC value, however. The MRI characteristics of these lesions are illustrated in Figures 1, 2, 3 and 4.

The AUC corresponding to ADC values between different mastitis subtypes are listed in Table 2. PDM and IAB exhibited the largest difference in ADC values $(\mathrm{AUC}=0.794)$, while GLM and IAB differed least in ADC value (AUC = $0.647)$. Specificity was also highest $(83.3 \%)$ for the comparison between PDM and IAB lesions.

The three groups did not vary significantly in non-mass internal enhancement $\left(\chi^{2}=7.462, P=0.113\right)$ or mass enhancement characteristics (shape: $\chi^{2}=5.884, P=0.208$; margin: $\chi^{2}=$ 5.887, $P=0.053$; internal enhancement: $\chi^{2}=0.398, P=0.819$ ). These results are shown in Table 3 . Likewise, there were no significant differences in lesion quadrant $(P=0.076)$, patient age $(P=0.445), \mathrm{T}_{2} \mathrm{WI}$ signal intensity $(P=0.477)$, DWI signal intensity $(P=0.129)$, or enhancement type $(P=0.119)$ among the three groups. These results are also shown in Table 4.

There was a significant difference in the distribution of non-mass enhancement among the three groups $\left(\chi^{2}=\right.$ 16.985, $P=0.03$, shown in Tables 3,5$)$. The most prevalent non-mass enhancement distribution types for PDM, GLM, and $\mathrm{IAB}$ were as follows: $\mathrm{PDM}=$ multiple-region $(7 / 15)$; GLM = diffuse (9/19); and IAB = regional (4/8). Examples of distribution types for these subtypes are shown in Figures 1, 2, 3 and 4. However, Fisher's exact test showed no significant difference in non-mass enhancement between any two groups: PDM vs. GLM ( $\left.\chi^{2}=9.847, P=0.210\right)$; PDM vs. IAB $\left(\chi^{2}=\right.$ 7.789, $P=0.061)$; and GLM vs. IAB $\left(\chi^{2}=1.484, P=0.484\right)$. Duct ectasia appeared in $58.8 \%(10 / 17)$ of PDM lesions, while micro-abscesses appeared in $62.5 \%$ (20/32) of GLM lsions. These results are shown in Table 3.

\section{Discussion}

In this study, we compared the morphological and functional characteristics of three subtypes of non-puerperal mastitis lesions primarily using DWI and DCE MRI. ADC values
Table 1 Comparison of ADC values for mastitis subtypes

\begin{tabular}{lllll}
\hline $\begin{array}{l}\text { ADC Values Comparison } \\
\text { of Subtypes }\end{array}$ & Mastitis Subtype & Case (n) & \multicolumn{2}{l}{ Alpha $=0.05$ Subset } \\
\cline { 4 - 5 } & & & 1 & 2 \\
\hline Student-Newman-Keuls test & PDM & 17 & & 1.4575208 \\
& IAB & 12 & 1.1053889 & \\
& GLM & 32 & 1.2438824 & 1.000 \\
\hline
\end{tabular}




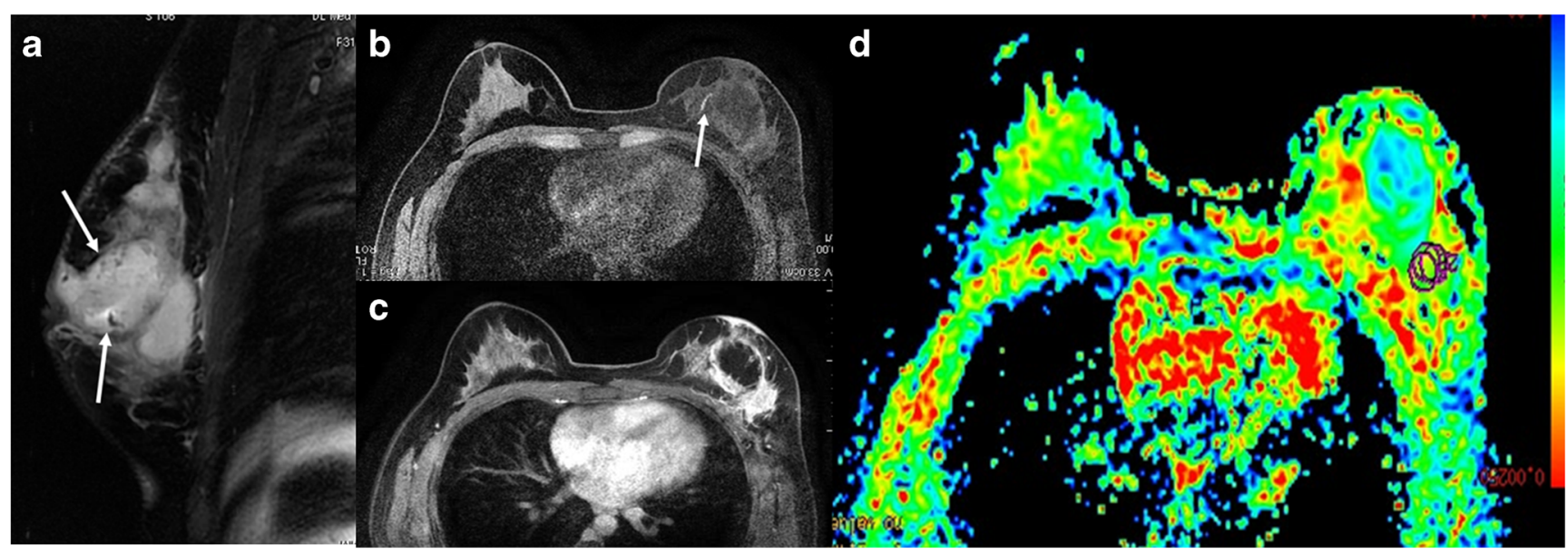

Fig. 1 (a-d): MRI presentation of 31-year-old patient with left-breast PDM: a) Sagittal $\mathrm{T}_{2}$ WI shows hyperintense signal of the lesion (arrow); b) Axial FSPGR shows linear hyperintense signal (indicative of duct

extrapolated from DWI have been previously used to differentiate between abscesses and cystic tumours in various regions of the body $[9,19]$. To date, however, the role of DWI in specifically differentiating between the major subtypes of non-puerperal mastitis has not been examined. Furthermore, the role of DCE MRI characteristics such as mass enhancement and non-mass enhancement in differentiating between mastitis subtypes has been limited.

Differentiating between PDM, GLM, and IAB during the non-puerperal period poses a significant diagnostic challenge due to the similar clinical presentations of these three subtypes of mastitis. Non-puerperal mastitis is commonly ectasia, arrow) within the lesion; c) DCE MRI illustrates non-mass, multiple-region heterogeneous enhancement of the lesion; d) ADC map gives ADC value of $1.597 \times 10^{-3} \mathrm{~mm}^{2} / \mathrm{s}\left(\mathrm{b}=800 \mathrm{~s} / \mathrm{mm}^{2}\right)$.

misdiagnosed, which can lead to incorrect treatment and a negative outcome for the patient $[3,4,6]$. Furthermore, many patients with non-puerperal mastitis present with one or several breast masses without other signs of inflammation. These masses often mimic inflammatory carcinoma both clinically and radiologically, making a definitive diagnosis of mastitis difficult [4]. It is of the utmost importance for breast specialists to both confirm the diagnosis of mastitis and determine the mastitis subtype before administering distinct therapeutic regimens, such as surgical excision, hormone therapy, or antibiotic treatment. While conventional MRI has been used as a potential imaging tool for differentiating mastitis from
Fig. 2 (a-d): MRI presentation of 43-year-old patient with rightbreast PDM: a) Axial FSPGR shows hypointense signal of the lesion; b) DCE MRI illustrates non-mass, diffuse clustered ring enhancing lesion; c) ADC map gives ADC value of $1.87 \times 10^{-3}$ $\left.\mathrm{mm}^{2} / \mathrm{s}\left(\mathrm{b}=800 \mathrm{~s} / \mathrm{mm}^{2}\right) ; \mathrm{d}\right)$ Timesignal intensity curve (TIC) shows persistent enhancement.

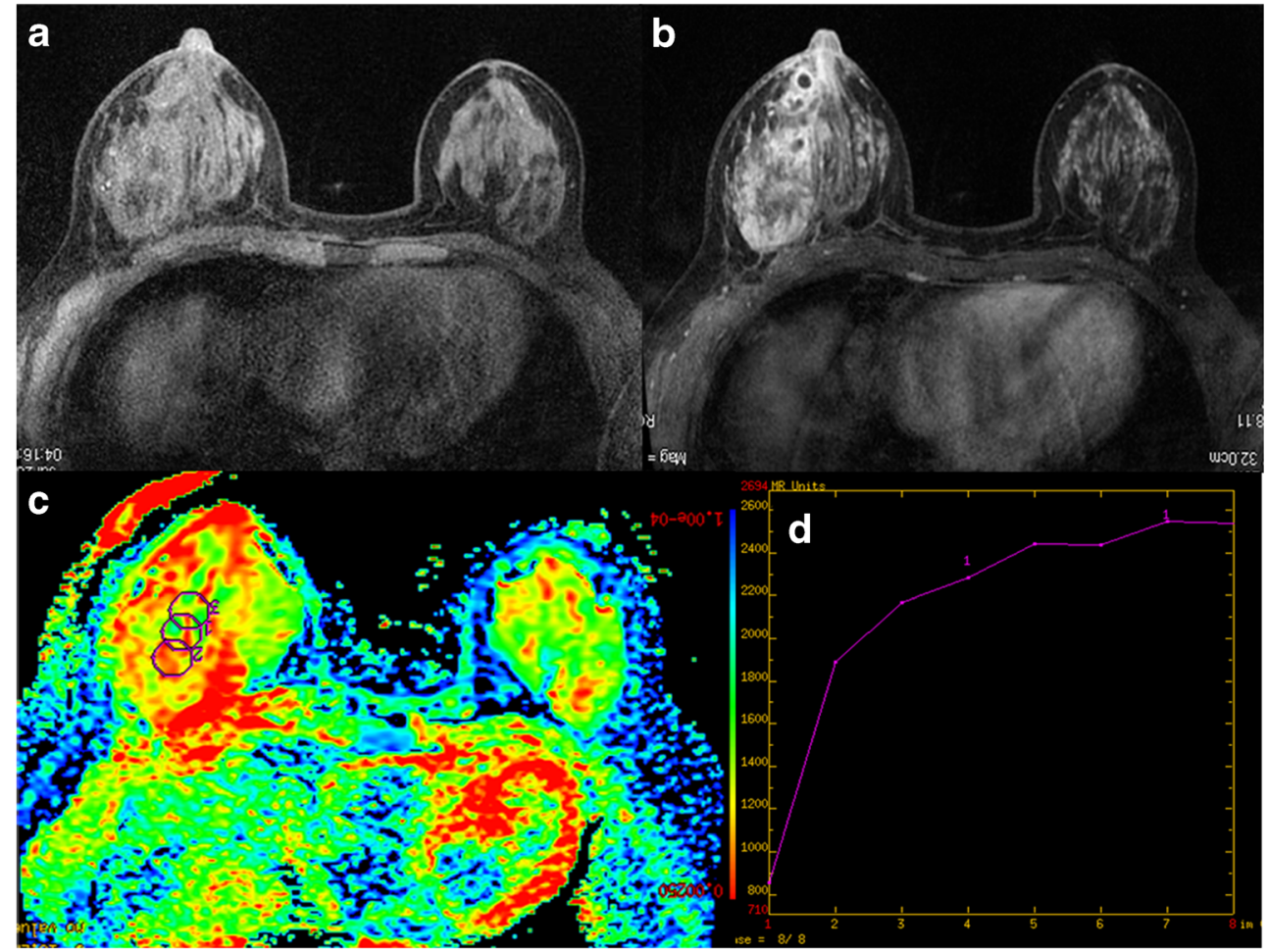


Fig. 3 (a-d): MRI presentation of 41-year-old patient with leftbreast GLM: a) Sagittal $T_{2}$ WI shows hyperintense signal of the lesion (arrow); b) DCE MRI illustrates mass enhancement of lesion with irregular shape, circumscribed margin, and heterogeneous internal enhancement; c) ADC map gives ADC value of $1.35 \times 10^{-3} \mathrm{~mm}^{2} / \mathrm{s}$ $\left.\left(\mathrm{b}=800 \mathrm{~s} / \mathrm{mm}^{2}\right) ; \mathrm{d}\right)$ Time-signal intensity curve (TIC) shows plateau enhancement.

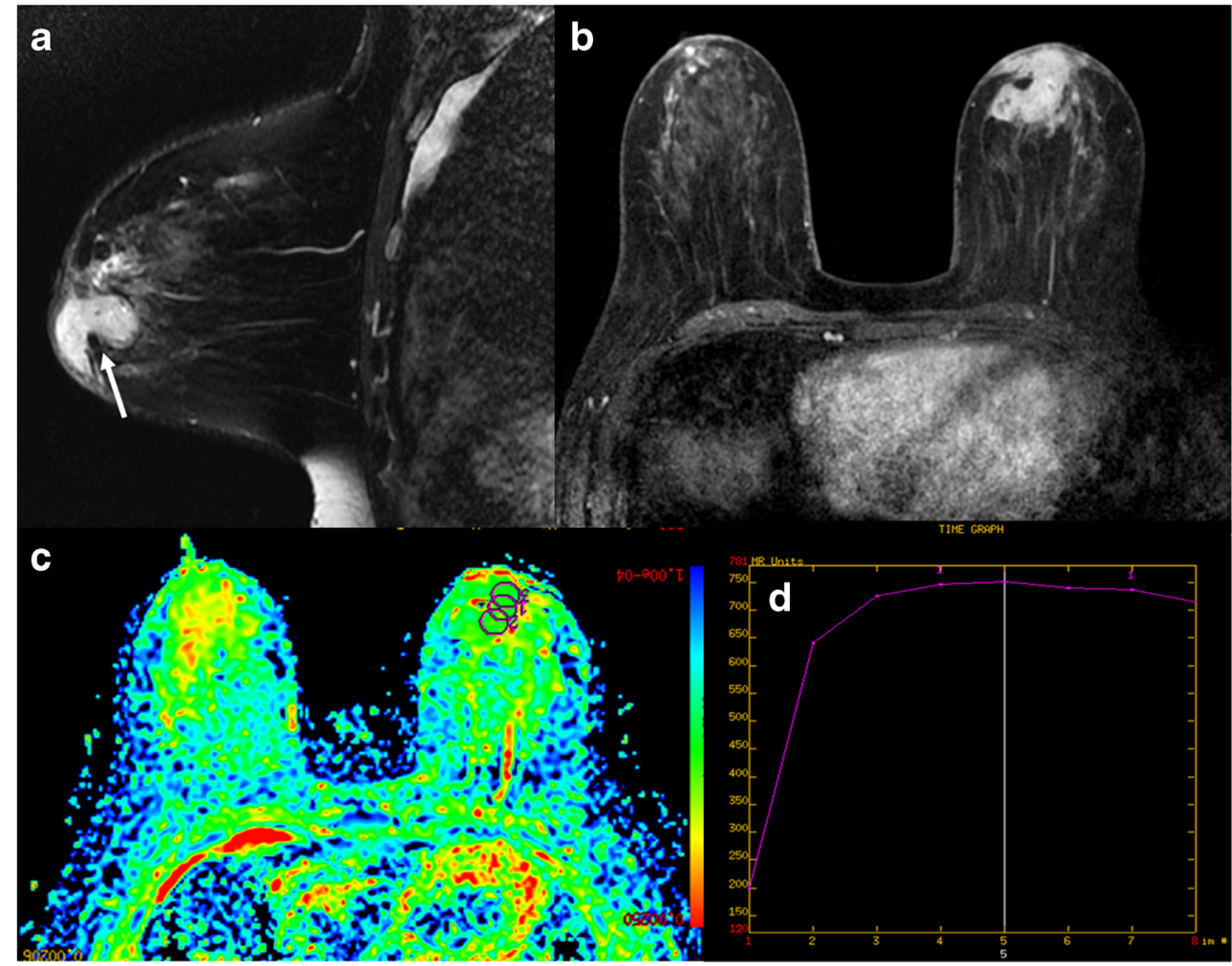

malignant breast lesions [7-9], it has not been effective in differentiating PDM from GLM, as PDM and GLM possess similar clinical and radiological characteristics, such as nonspecific enhancement, skin thickening, diffuse oedema, and abnormal nipple configuration [8]. Additionally, IAB may mimic non-infectious mastitis on conventional MRI, especially when the clinical symptoms of IAB are atypical and the abscess has not yet formed [8, 9].
PDM, which most commonly presents in areas surrounding the areola $[1,4]$, is characterised by the infiltration of plasma cells and lymphocytes, and the presence of these cells is a hallmark of PDM pathologically. Previous studies [4, 12] have hypothesised that duct ectasia, followed by plasma cell-induced inflammation, plays an integral role in the pathophysiology of PDM. Lactiferous duct occlusion may lead to fluid retention and subsequent lipid spillover, thereby precipitating an influx of plasma cells and
Fig. 4 (a-d): MRI presentation of 42-year-old patient with rightbreast IAB: a) DCE MRI illustrates non mass enhancement of lesion with regional and heterogeneous enhancement; $b$ ) DWI $\left(b=800 \mathrm{~s} / \mathrm{mm}^{2}\right)$ shows hyperintense signal of the lesion; c) ADC map gives ADC value of $1.09 \times 10^{-3} \mathrm{~mm}^{2} / \mathrm{s}$; d) Time-signal intensity curve (TIC) shows persistent enhancement.

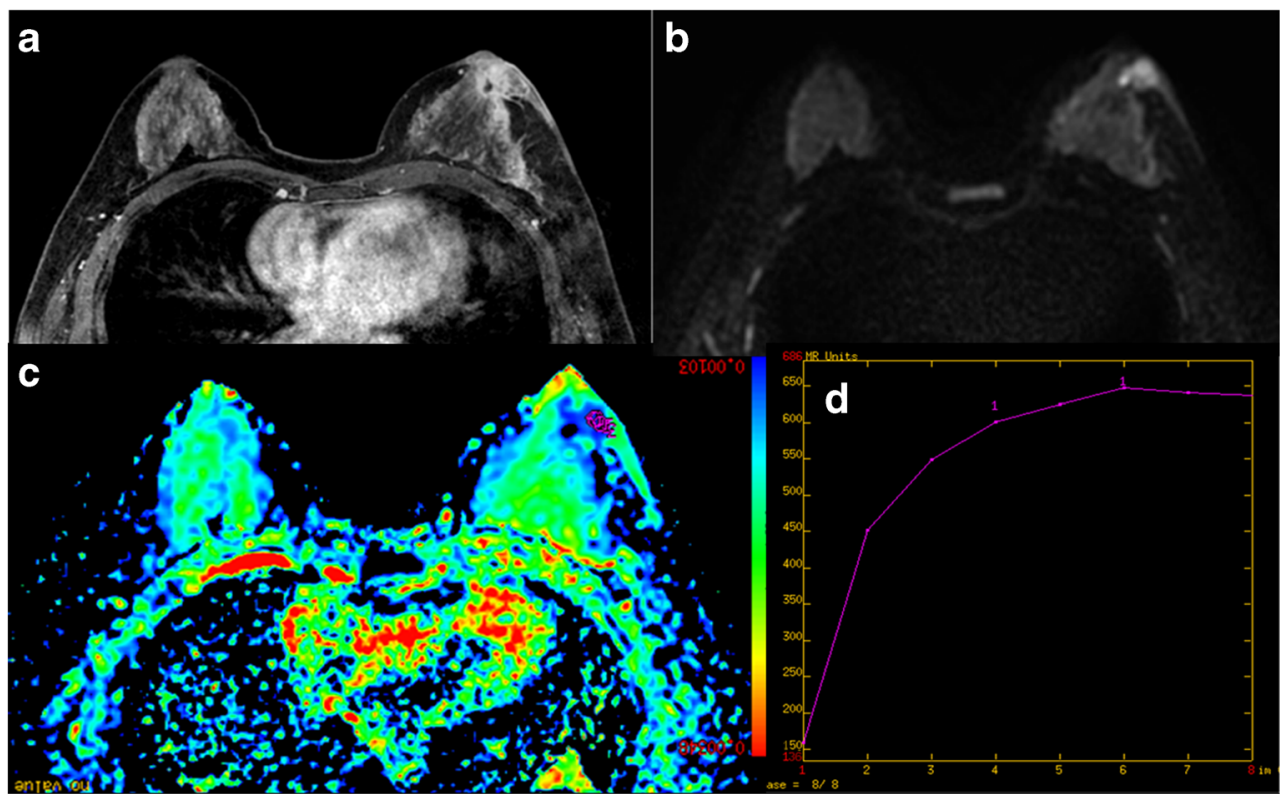


Table 2 AUC, diagnostic threshold, and sensitivity and specificity of ADC values for mastitis subtypes

\begin{tabular}{llllc}
\hline Variables (ADC Value) & AUC & Cut-off Value & Sensitivity & Specificity \\
\hline PDM and IAB & 0.794 & $1.348 \times 10^{-3} \mathrm{~mm}^{2} / \mathrm{s}$ & 0.813 & 0.833 \\
PDM and GLM & 0.733 & $1.328 \times 10^{-3} \mathrm{~mm}^{2} / \mathrm{s}$ & 0.844 & 0.647 \\
IAB and GLM & 0.647 & $1.125 \times 10^{-3} \mathrm{~mm}^{2} / \mathrm{s}$ & 0.706 & 0.667 \\
\hline
\end{tabular}

associated inflammation in the surrounding tissue. Duct ectasia was observed in $58.8 \%$ (10/17) of the PDM patients in our study. Still, PDM lesions exhibited the largest ADC value $[(1.558 \pm$ $0.34) \times 10^{-3} \mathrm{~mm}^{2} / \mathrm{s}$ ] in our study, suggesting that the inflammation associated with this mastitis subtype has, in general, less restrictive diffusion than that associated with GLM or IAB.

GLM lesions can affect any quadrant of the breast $[1,4]$ and have been shown to exhibit both periductal inflammation $[20,21]$ and the presence of microabscesses, which result in a decrease in free water diffusion as compared to healthy parenchyma [13]. In our study, microabscesses appeared in $62.5 \%$ (20/32) of GLM lesions. Minor periductal inflammation was also evident in the majority of our GLM cases, likely contributing to a significantly lower ADC value $\left[(1.244 \pm 0.32) \times 10^{-}\right.$ $\left.{ }^{3} \mathrm{~mm}^{2} / \mathrm{s}\right]$ than that for PDM $\left[(1.558 \pm 0.34) \times 10^{-3} \mathrm{~mm}^{2} / \mathrm{s}\right]$. In addition to periductal inflammation and microabscesses, the

Table 3 Lesion MR enhancement characteristics stratified by mastitis subtype (n)

\begin{tabular}{|c|c|c|c|c|c|}
\hline & GLM & IAB & PDM & $x^{2}$ & $P$-value \\
\hline Non-mass & 19 & 8 & 15 & & \\
\hline Enhancement Distribution & & & & 16.985 & $0.03^{*}$ \\
\hline Focal & 0 & 0 & 0 & & \\
\hline Linear & 3 & 0 & 0 & & \\
\hline Segmental & 3 & 0 & 0 & & \\
\hline Regional & 1 & 4 & 4 & & \\
\hline Multiple regions & 3 & 2 & 7 & & \\
\hline Diffuse & 9 & 2 & 4 & & \\
\hline Internal Enhancement & & & & 7.462 & 0.113 \\
\hline Homogenous & 3 & 0 & 0 & & \\
\hline Heterogeneous & 8 & 6 & 12 & & \\
\hline Clustered Ring & 8 & 2 & 3 & & \\
\hline Mass & 13 & 4 & 2 & & \\
\hline Shape & & & & 5.884 & 0.208 \\
\hline Oval & 2 & 1 & 0 & & \\
\hline Round & 1 & 2 & 0 & & \\
\hline Irregular & 10 & 1 & 2 & & \\
\hline Margin & & & & 5.887 & 0.053 \\
\hline Circumscribed & 4 & 4 & 1 & & \\
\hline Irregular & 9 & 0 & 1 & & \\
\hline Internal Enhancement & & & & 0.398 & 0.819 \\
\hline Heterogeneous & 4 & 1 & 1 & & \\
\hline Rim Enhancement & 9 & 3 & 1 & & \\
\hline
\end{tabular}

${ }^{*} P<0.05$ was considered statistically significant. presence of blood or other viscous fluids in dilated lactiferous ducts can contribute to the restricted diffusion (and thus lower ADC value) seen in GLM lesions. Furthermore, these viscous fluids may mimic malignancies such as ductal cancers on DWI $[10,11,16]$, adding to the challenge of differentiating between breast cancers and mastitis lesions.

Lastly, IAB lesions, which can be located centrally or peripherally (lesions were $50 \%$ central and $50 \%$ peripheral in our study), are characterised by the accumulation of massive inflammatory cells, microorganisms, large protein molecules, necrotic tissue, and cellular debris, all of which restrict free water diffusion [8-10]. Pathologically, squamous metaplasia of the cuboidal epithelium leads to the formation of keratin plugs, acute central inflammation, and the accumulation of cellular debris. Secondary infection ensues, leading to stagnation and subsequent abscess formation [5, 22, 23]. This pathophysiological mechanism of IAB culminates in a gross lack of free water diffusion, illustrated in our study by the hyperintensity observed on DWI and the low ADC value $\left[(1.105 \pm 0.44) \times 10^{-3} \mathrm{~mm}^{2} / \mathrm{s}\right]$ as compared to PDM and GLM. While the ADC was an effective parameter for differentiating between IAB and PDM, we did not observe a significant difference in $\mathrm{ADC}$ value between IAB and GLM. It is possible that the similar manifestations of the microabscesses in GLM and the larger IAB lesions contributed to this lack of ADC difference, though future studies are necessary. Nevertheless, the ADC values derived from DWI have been effective in differentiating IAB lesions from cystic or necrotic tumours in various regions of the body, such as the brain and liver [9, 24-27]. Malignancies generally appear hypointense on both DWI and $\mathrm{T}_{2}$ WI, while benign lesions such as nonpuerperal mastitis show a hyperintense signal [8, 9]. Unal et al. [9] attribute this intensity difference on DWI to the different physical and biochemical contents of the lesions. Most notably, cystic and necrotic tumours contain significantly fewer inflammatory cells and cellular debris than IAB lesions, allowing for these malignancies to be differentiated from mastitis on DWI.

In sum, ADC values, which reflect the degree of water molecule diffusion through tissue, proved most effective for differentiating between mastitis subtypes in our study.

When analysing each type of lesion individually, PDM lesions exhibit the highest ADC values of the three mastitis subtypes. Furthermore, our results illustrate significant differences in ADC values among these three subtypes, as well as between members of two subtype pairs (PDM vs. GLM and PDM vs. IAB). PDM and IAB lesions differed most in ADC value, while GLM and IAB lesions differed least. We suspect that the 
Table 4 Patient age and lesion MR characteristics stratified by mastitis subtype (n)

\begin{tabular}{|c|c|c|c|c|c|}
\hline & GLM & $\mathrm{IAB}$ & PDM & $x^{2}$ & $P$-value \\
\hline Patient Age (y) & $44.47 \pm 14.68$ & $41.33 \pm 18.79$ & $41.34 \pm 12.71$ & 1.617 & 0.445 \\
\hline Lesion Quadrant & & & & 11.415 & 0.076 \\
\hline Lateral-Superior & 6 & 4 & 0 & & \\
\hline Interior-Superior & 9 & 4 & 2 & & \\
\hline Lateral-Inferior & 11 & 2 & 11 & & \\
\hline Interior-Inferior & 6 & 2 & 4 & & \\
\hline $\begin{array}{l}\text { T2WI Signal } \\
\text { Intensity }\end{array}$ & & & & 3.504 & 0.477 \\
\hline Isointensity & 6 & 0 & 2 & & \\
\hline Hypointensity & 2 & 2 & 2 & & \\
\hline Hyperintensity & 24 & 10 & 13 & & \\
\hline DWI signal intensity & & & & 7.138 & 0.129 \\
\hline Isointensity & 10 & 0 & 4 & & \\
\hline Hypointensity & 4 & 1 & 4 & & \\
\hline Hyperintensity & 18 & 11 & 9 & & \\
\hline
\end{tabular}

different pathological states of PDM (duct ectasia), GLM (microabscesses), and IAB (gross restriction of free water diffusion) could be responsible for the differences in ADC values shown in Table 1. More specifically, the degree of inflammatory cell infiltration, tissue necrosis, and tissue regeneration primarily contributed to the different ADC values obtained, and aided us in our development of these diagnostic thresholds. Still, further studies on the DWI characteristics of mastitis lesions are warranted in order to obtain conclusive data.

It is known that DWI signals can be affected by $\mathrm{T} 2$ shine effects, while ADC values are not. In this study, we calculated mean ADC values according to the most enhanced lesion areas on DCE-MRI The ADC values were measured excluding the completely necrotic or cystic components and can reflect the characteristic of the mastitis lesions. As shown in our study, calculating ADC values using this approach can greatly aid in distinguishing the different types of non-puerperal mastitis, with the added benefits of avoiding the influences of $\mathrm{T} 2$ shine effects. Consistent with previous mastitis imaging studies [3, 17, 28], all mastitis lesions in our study exhibited a hyperintense signal on T2WI, with no significant difference in T2WI or DWI signal intensity among the three groups. We speculated that the fluidlike, high-protein nature of these mastitis lesions and the

Table 5 Enhancement types stratified by mastitis subtype (\%)

\begin{tabular}{llcc}
\hline Subtype & Cases & Non-Mass Enhancement & Mass Enhancement \\
\hline GLM & 32 & $19(59.4)$ & $13(40.6)$ \\
IAB & 12 & $8(66.7)$ & $4(33.3)$ \\
PDM & 17 & $15(88.2)$ & $2(11.8)$ \\
Total & 61 & $42(68.9)$ & $19(31.1)$ \\
\hline
\end{tabular}

similarities between the microabscesses seen in GLM and the IAB lesions likely produced similar signal intensities.

Research examining the DCE MRI characteristics of mastitis has been limited $[3,4,13]$. In our study, there was no significant difference in the distribution of mass enhancement or non-mass internal enhancement among the three mastitis subtypes. We did find a significant difference in the distribution of non-mass enhancement among the three groups, though no two subtypes were significantly different from each other when compared individually. Nevertheless, we believe there is value in utilising the qualitative DCE MRI characteristics of each mastitis subtype. The most common distribution types for PDM, GLM, and IAB were multiple-region, diffuse, and regional, respectively. It is possible that these distribution types could complement the ADC values obtained from DWI to more accurately characterise the mastitis subtypes.

While our results offer promising potential for imaging various subtypes of mastitis, there are some limitations to our study. Firstly, it was performed retrospectively, and our sample size was relatively small. In addition, we did not analyse the kinetic curves of the different subtypes of mastitis. In future studies, a comparative analysis of DCE MRI versus DWI should be performed.

\section{Conclusions}

Our results illustrate new methods of differentiating between various mastitis lesions using DWI and DCE MRI. The ADC value derived from DWI for each subtype of mastitis was especially useful for differentiating PDM from GLM and IAB lesions. This parameter has promising potential as a means of further investigating the characteristics of mastitis lesions on DWI. Additionally, the distribution of non-mass enhancement 
on DCE MRI offers a new lens into the imaging characteristics of mastitis. With further examination of DWI and DCE MRI in future studies, these imaging modalities could reduce the time between clinical presentation and definitive diagnosis and treatment, ultimately improving patient outcomes.

Funding The authors state that this work has not received any funding.

\section{Compliance with ethical standards}

Guarantor The scientific guarantor of this publication is Ailian Liu.

Conflict of interest The authors of this manuscript declare no relationships with any companies, whose products or services may be related to the subject matter of the article.

Statistics and biometry No complex statistical methods were necessary for this paper.

Informed consent The requirement for informed consent was waived for this study due to its retrospective design.

Ethical approval This retrospective study was approved by the Institutional Ethics Committee of the First Affiliated Hospital of Dalian Medical University (Dalian, China) and was performed in accordance with the ethical guidelines of the Declaration of Helsinki.

\section{Methodology \\ - retrospective \\ - randomised controlled trial \\ - performed at one institution}

Open Access This article is distributed under the terms of the Creative Commons Attribution 4.0 International License (http:// creativecommons.org/licenses/by/4.0/), which permits unrestricted use, distribution, and reproduction in any medium, provided you give appropriate credit to the original author(s) and the source, provide a link to the Creative Commons license, and indicate if changes were made.

\section{References}

1. Kasales CJ, Han B, Smith JS Jr, Chetlen AL, Kaneda HJ, Shereef S (2014) Nonpuerperal mastitis and subareolar abscess of the breast. AJR Am J Roentgenol 202:133-139

2. Peters F, Schuth W (1989) Hyperprolactinemia and nonpuerperal mastitis (duct ectasia). JAMA 261:1618-1620

3. Liu H, Peng W (2011) Morphological manifestations of nonpuerperal mastitis on magnetic resonance imaging. J Magn Reson Imaging 33:1369-1374

4. Tan H, Li R, Peng W, Liu H, Gu Y, Shen X (2013) Radiological and clinical features of adult non-puerperal mastitis. Br J Radiol 86:1024

5. Bosma MS, Morden KL, Klein KA, Neal CH, Knoepp US, Patterson SK (2016) Breast imaging after dark: patient outcomes following evaluation for breast abscess in the emergency department after hours. Emerg Radiol 23:29-33

6. Dursun M, Yilmaz S, Yahyayev A et al (2012) Multimodality imaging features of idiopathic granulomatous mastitis: outcome of 12 years of experience. Radiol Med 117:529-538

7. Ferris-James DM, Iuanow E, Mehta TS, Shaheen RM, Slanetz PJ (2012) Imaging approaches to diagnosis and management of common ductal abnormalities. Radiographics 32:1009-1030
8. Renz DM, Baltzer PA, Bottcher J et al (2008) Magnetic resonance imaging of inflammatory breast carcinoma and acute mastitis: a comparative study. Eur Radiol 18:2370-2380

9. Unal OI, Koparan HI, Avcu S et al (2011) The diagnostic value of diffusion-weighted magnetic resonance imaging in soft tissue abscesses. Eur J Radiol 77:490-494

10. Orguc S, Basara I, Coskun T (2012) Diffusion-weighted MR imaging of the breast: comparison of apparent diffusion coefficient values of normal breast tissue with benign and malignant breast lesions. Singapore Med 53:737-743

11. Ramírez-Galván YA, Cardona-Huerta S, Ibarra-Fombona E, Elizondo-Riojas G (2015) Apparent diffusion coefficient (ADC) value to evaluate BI-RADS 4 breast lesions: correlation with pathological findings. Clin Imaging 39:51-55

12. Cheng L, Reddy V, Solmos G et al (2015) Mastitis, a Radiographic, Clinical, and Histopathologic Review. Breast J 21:403-409

13. Aslan H, Pourbagher A, Colakoglu T (2015) Idiopathic granulomatous mastitis: magnetic resonance imaging findings with diffusion MRI. Acta Radiol. https://doi.org/10.1177/0284185115609804

14. Imamura T, Isomoto I, Sueyoshi E et al (2010) Diagnostic performance of ADC for non-mass-like breast lesions on MR imaging. Magn Reson Med Sci 9:217-225

15. Partridge SC, Mullins CD, Kurland BF et al (2010) Apparent diffusion coefficient values for discriminating benign and malignant breast MRI lesions: effects of lesion type and size. Am J Roentgenol 194:1664-1673

16. Marini C, Iacconi C, Giannelli M, Cilotti A, Moretti M, Bartolozzi C (2007) Quantitative diffusion weighted MR imaging in the differential diagnosis of breast lesion. Eur Radiol 17:2646-2655

17. Handa P, Leibman AJ, Sun D, Abadi M, Goldberg A (2014) Granulomatous mastitis: changing clinical and imaging features with image-guided biopsy correlation. Eur Radiol 24:2404-2411

18. Pinker K, Bickel H, Helbich TH et al (2013) Combined contrastenhanced magnetic resonance and diffusion-weighted imaging reading adapted to the "Breast Imaging Reporting and Data System" for multiparametric 3-T imaging of breast lesions. Eur Radiol 23:17911802

19. Koh DM, Collins DJ (2007) Diffusion-weighted MRI in the body: applications and challenges in oncology. AJR Am J Roentgenol 188:1622-1635

20. Erhan Y, Veral A, Kara E et al (2000) A clinicopathologic study of a rare clinical entity mimicking breast carcinoma: idiopathic granulomatous mastitis. Breast 9:52-56

21. Heer R, Shrimankar J, Griffith CD (2003) Granulomatous mastitis can mimic breast cancer on clinical, radiological or cytological examination: a cautionary tale. Breast 12:283-286

22. Lannin DR (2004) Twenty-two year experience with recurring subareolar abscess and lactiferous duct fistula treated by a single breast surgeon. Am J Surg 188:407-410

23. Versluijs-Ossewaarde FN, Roumen RM, Goris RJ (2005) Subareolar breast abscesses: characteristics and results of surgical treatment. Breast J 11:179-182

24. Le Bihan D, Turner R, Douek P, Patronas N (1992) Diffusion MR Imaging: clinical applications. AJR Am J Roentgenol 159:591-599

25. Ebisu T, Tanaka C, Umeda M et al (1996) Discrimination of brain abscess from necrotic cystic tumors by diffusion-weighted echo planar imaging. Magn Reson Imaging 14:1113-1116

26. Castillo M, Mukherji KS (2000) Diffusion-weighted imaging of intracranial lesions. Semin Ultrasound CT MR 21:405-415

27. Demir OI, Obuz F, Sagol O, Dicle O (2007) Contribution of diffusion-weighted MRI to the differential diagnosis of hepatic masses. Diagn Interv Radiol 13:81-86

28. Gautier N, Lalonde L, Tran-Thanh D et al (2013) Chronic granulomatous mastitis: Imaging, pathology and management. Eur J Radiol $82: 165-175$ 